LA-UR- $95=754$

Title:

OFF-SPECULAR SCATTERING IN NEUTRON REFLECTOMETRY
Author(s):

Submitted to:

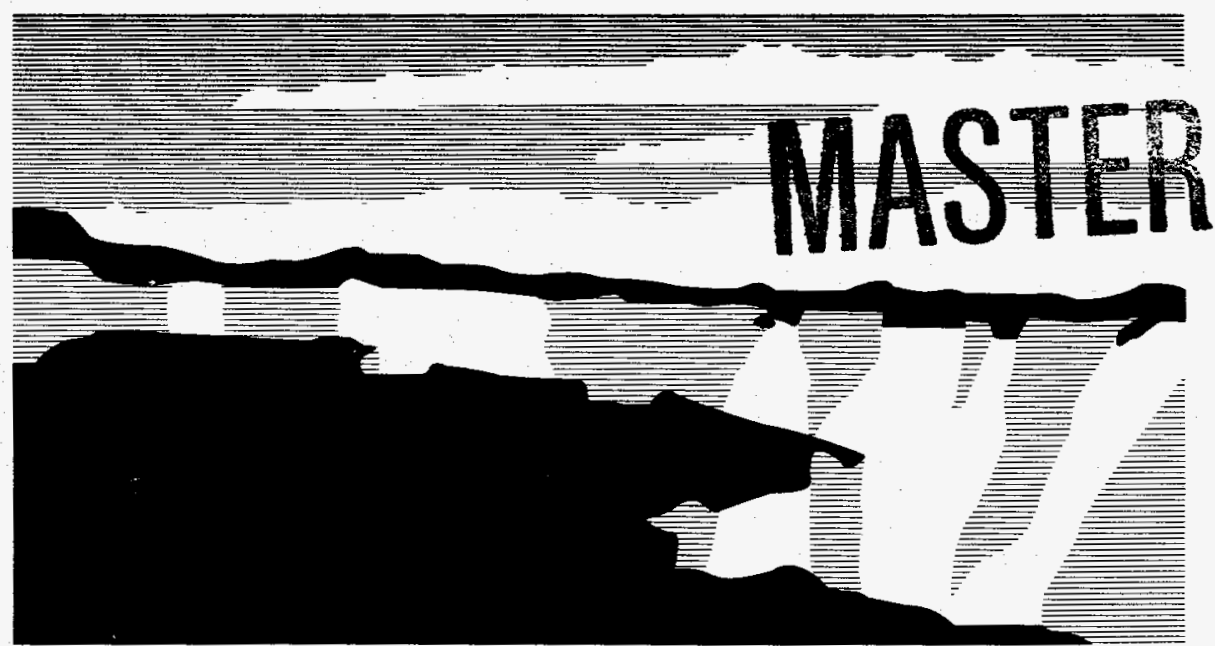

RECEIVED

MAR 101995

OSTI

Invited talk at "Workshop on Neutron Applications

in Materials Science and Engineering," Chalk River, Canada, 17. Aug 94 


\section{DISCLAIMER}

This report was prepared as an account of work sponsored by an agency of the United States Government. Neither the United States Government nor any agency thereof, nor any of their employees, make any warranty, express or implied, or assumes any legal liability or responsibility for the accuracy, completeness, or usefulness of any information, apparatus, product, or process disclosed, or represents that its use would not infringe privately owned rights. Reference herein to any specific commercial product, process, or service by trade name, trademark, manufacturer, or otherwise does not necessarily constitute or imply its endorsement, recommendation, or favoring by the United States Government or any agency thereof. The views and opinions of authors expressed herein do not necessarily state or reflect those of the United States Government or any agency thereof. 


\section{DISCLAIMER}

Portions of this document may be illegible in electronic image products. Images are produced from the best available original document. 
PLEASE NOTE: Some figures are on the end sheets, not in the text itself. 


\title{
Off-Specular Scattering in Neutron Reflectometry
}

\author{
by \\ Roger Pynn, Shenda M. Baker, Greg Smith and Mike Fitzsimmons \\ Manuel Lujan Jr. Neutron Scattering Center, Los Alamos National Laboratory, \\ Los Alamos, NM 87545, U.S.A.
}

\begin{abstract}
$\underline{\text { Abstract }}$
When neutrons are scattered at small angles from planar, laterally homogeneous, stratified media, only specular (mirror like) reflection is observed. Sample inhomogeneities, such as interfacial roughness or voids, give rise to off-specular scattering which has been observed in many experiments with neutrons and $x$-rays. The easiest way to describe this scattering theoretically is based on the distorted-wave Born approximation (DWBA), which uses the neutron wavefunctions that describe reflection from a smooth surface as the basis functions for perturbation theory. From the DWBA one may obtain a number of qualitative results which are supported by experiment. Examples include the Yoneda fringes observed in reflection experiments with microscopically rough surfaces and the constant- $\mathrm{q}_{z}$ fringes observed for multilayers with correlated, rough interfaces. One must, however, use the DWBA with care. When the correlation range within the reflecting interfaces is large - for example, when a surface is composed of misoriented facets - the approximation breaks down. Some authors have also reported a lack of quantitative agreement between versions of the DWBA calculations and the scattering observed with microscopically rough surfaces. A remarkable feature of neutron (or $\mathrm{x}$-ray) reflectometry is the length scales that are probed within reflecting surfaces. These range from a few hundred Ångstrøms up to several microns, allowing neutron scattering to probe objects of a size normally visible by optical microscopy! The intent of this paper is to provide a simple description of scattering from rough surfaces that is accessible to a wide audience. Mathematical completeness is sacrificed in favor of intuitive arguments and experimental examples.
\end{abstract}

Introduction

Let us consider the simple reflectometry experiment depicted in Figure 1. Incident neutrons of wavevector $\mathbf{k}_{1}$ are specularly reflected to wavevector $\mathbf{k}_{2}$. In this case, the grazing angle of incidence, $\theta_{1}$, is equal to the grazing angle of reflection, $\theta_{2}$. Provided $k_{1} \sin \theta_{1}$ is large enough (i.e. greater than the critical wavevector for the reflecting material), some of the beam is transmitted as wavevector $\mathbf{k}_{1}{ }_{1}$. Because the surface is rough, however, there is diffuse (i.e. off-specular) scattering both above and below the horizon (i.e. for $\theta_{2}>0$ and $\left.\theta_{2}<0\right)$.

Although it is not depicted in Figure 1, off-specular scattering can also result from imperfections within the scattering medium. In this case, the transmitted beam is scattered through small angles and may be observed either above or below the horizon. We will not consider this case in detail here, although it is important, for example, in experiments where the medium below the surface is a complex fluid which is ordered when it flows past a solid material above the interface [1]. 


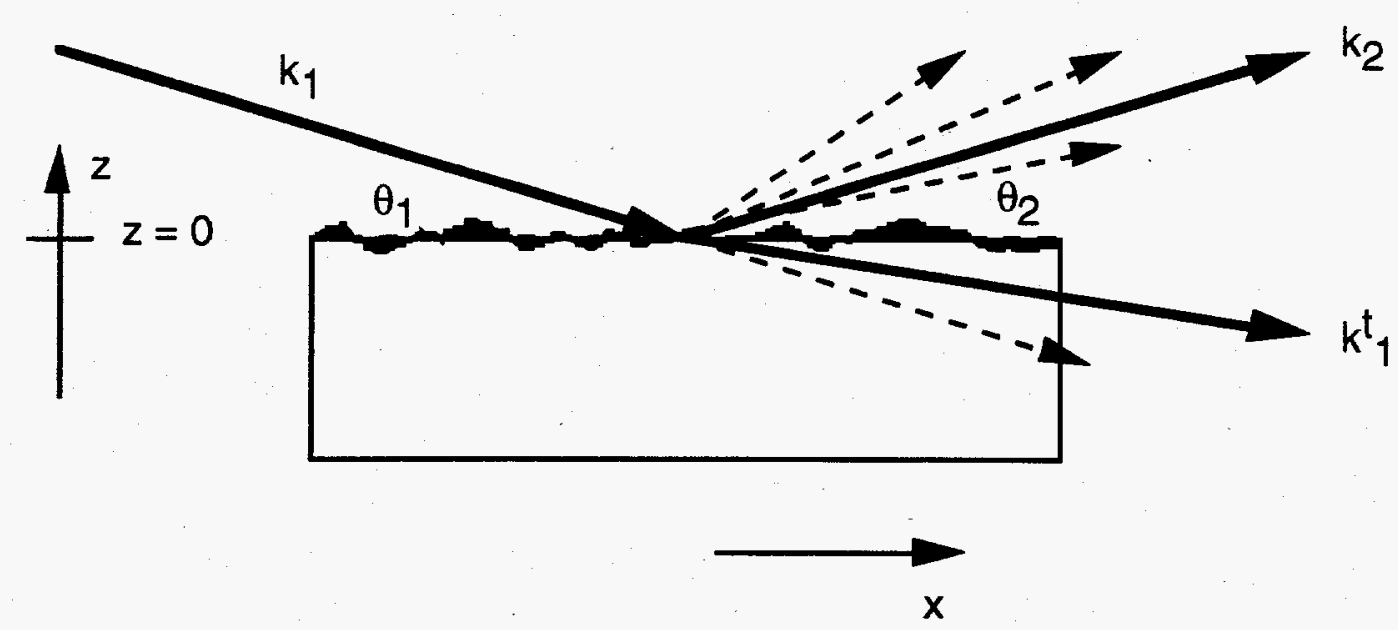

Figure 1: A neutron reflection experiment with a rough sample. The bold arrows represent the incident, specularly reflected and transmitted neutrons. The dashed arrows show diffuse scattering from the rough surface. The region between the rough surface and an average, smooth surface (referred to as the ideal surface) is shaded.

The mathematical description of the diffuse scattering from one or more rough interfaces is fairly complex. Indeed, in the case of radar waves reflected from rough terrain, it has been the subject of a fairly long book by Beckmann and Spizzichino [2], many of whose ideas are applicable to the problem of neutron (or x-ray) reflectometry. Rather than present detailed derivations of scattering theory in this paper, we will try motivate equations in a more heuristic fashion and present examples.

\section{The Distorted Wave Born Approximation}

The traditional way of calculating the scattering of neutrons in diffraction experiments is to use the Born approximation in conjunction with the Fermi pseudopotential, parametrised by the atomic scattering length. In this approximation, one imagines a plane wave incident on a weak scatterer and uses simple perturbation theory - Fermi's golden rule - to calculate the scattered intensity. The method gives very accurate answers when it is applied to neutron diffraction or small angle scattering, but we might imagine that it would not work well for reflection geometry, especially close to the critical wavevector. In the latter case, most of the incident beam is specularly reflected and only a small fraction transmitted - in contrast to situations in which the Born approximation is adequate, where most of the neutrons are not deviated by the scattering potential.

A better way of doing perturbation theory for a reflecting surface is to use as a basis the wavefunctions for a perfectly smooth surface, under the assumption that surface roughness will only cause small corrections to specular scattering. The wave functions in question are the incident and specularly reflected plane waves above the reflecting surface (for $z>0$ ) and the transmitted plane wave below the surface. We can write these as:

$$
\begin{array}{ll}
\psi_{1 \mathrm{i}}^{(+)}(\mathbf{r})=\exp \left[\mathrm{i}\left(\mathrm{k}_{1 \mathrm{x}} \mathrm{x}+\mathrm{k}_{1 \mathrm{y}} \mathrm{y}\right)\right]\left[\exp \left(-\mathrm{ik} \mathrm{k}_{1 \mathrm{z}} \mathrm{z}\right)+\mathrm{R}_{\mathrm{i}}\left(\mathrm{k}_{1 \mathrm{z}}\right) \exp \left(\mathrm{ik}_{1 \mathrm{z}} \mathrm{z}\right)\right] & \text { for } \mathrm{z}>0 \\
\psi_{1 \mathrm{i}}^{(+)}(\mathbf{r})=\exp \left[\mathrm{i}\left(\mathrm{k}_{1 \mathrm{x}} \mathrm{x}+\mathrm{k}_{1 \mathrm{y}} \mathrm{y}\right)\right] \mathrm{T}_{\mathrm{i}}\left(\mathrm{k}_{1 \mathrm{z}}\right) \exp \left(-\mathrm{ik}_{1 \mathrm{z}}^{\mathrm{t}} \mathrm{z}\right) & \text { for } \mathrm{z}<0
\end{array}
$$

where $R_{i}$ and $T_{i}$ are the reflection and transmission coefficients of the ideal (i.e. smooth) surface which are given by the usual Fresnel expressions discussed by Majkrzak at this school [3]. 
An important fact that we will make use of shortly is that the wavefunction in eqn (1) and its first derivative are continuous at the reflecting surface (i.e. at $z=0$ ). This means that, close to the surface and above it, we can replace the wavefunction on the first line of eqn (1) with that from the second line without making much error.

To calculate the diffuse scattering due to surface roughness, we do a perturbation calculation using the wavefunctions in eqn (1) and a potential that corresponds to the difference between a sample with an ideal flat surface and one with a rough surface. This is shown by the shaded region in Figure 1. Where the rough surface is above the ideal surface we add the potential of the scattering medium and where the rough surface is below we subtract that potential. The scattering potential in question is just $\left(\mathrm{h}^{2} \mathrm{~N} b\right) /(2 \pi \mathrm{m})$, where $\mathrm{Nb}$ is the scattering length density of the medium, $h$ is Plank's constant and $m$ the neutron mass. Making use of the continuity of the wavefunction at the ideal surface, we can then write the perturbation term as:

$$
\left\langle\psi_{2 \mathrm{i}}^{(-)}|\mathrm{V}| \psi_{1 \mathrm{i}}^{(+)}\right\rangle=\frac{h^{2} \mathrm{Nb}}{2 \pi \mathrm{m}} \int \mathrm{dx} d y d z \mathrm{e}^{-\mathrm{i}\left(\mathrm{q}_{\mathrm{x}} \mathrm{x}+\mathrm{q}_{\mathrm{y}} \mathrm{y}\right)} \mathrm{T}_{\mathrm{i}}\left(\mathrm{k}_{1}\right) \mathrm{T}_{\mathrm{i}}\left(\mathrm{k}_{2}\right) \mathrm{e}^{-\mathrm{i}\left(\mathbf{k}_{\mathrm{z}}^{\mathrm{t}}+\mathbf{k}_{\mathrm{zz}}^{\mathrm{t}}\right) \mathrm{z}}
$$

where the integral extends over the shaded region of Figure 1. Note that this expression differs from the normal Born approximation by the inclusion of the two transmission functions and by the fact that the $\mathrm{z}$-dependent exponential involves wavevectors evaluated within the reflecting medium. These are defined by:

$$
\mathrm{k}_{1 \mathrm{z}}^{\mathrm{t}}=\left(\mathrm{k}_{1 \mathrm{z}}^{2}-\mathrm{k}_{\mathrm{c}}^{2}\right)^{1 / 2}=\left(\mathrm{k}_{1 \mathrm{z}}^{2}-4 \pi \mathrm{Nb}\right)^{1 / 2}
$$

In the traditional Born approximation, refractive corrections such as those embodied in eqn (3) are omitted.

The diffuse scattering cross section is proportional to the modulus of eqn (2) squared and can be written as:

$$
\left(\frac{d \sigma}{d \Omega}\right)_{\text {diffuse }}=N^{2} b^{2} L_{x} L_{y}\left|T_{i}\left(k_{1}\right)\right|^{2}\left|T_{i}\left(k_{2}\right)\right|^{2} S\left(q_{x}, q_{y}, q_{z}^{t}\right)
$$

where

and

$$
\begin{gathered}
S\left(q_{x}, q_{y}, q_{z}^{t}\right)=\frac{1}{\mid q_{2}^{t t^{2}}} e^{-\left(q_{z}^{t^{2}}+q_{z}^{t^{* 2}}\right) \sigma^{2} / 2} \int d x d y e^{i\left(q_{x} x+q_{y} y\right)\left(e^{d} q_{2}^{2} C(x, y)-1\right)} \\
q_{z}^{t}=k_{1 z}^{t}-k_{2 z}^{t}
\end{gathered}
$$

$$
C(x, y)=\langle z(x, y) z(0,0)\rangle
$$

Eqn (7) is just the height-height correlation function for the surface, whose roughness is assumed to be Gaussian with a standard deviation $\sigma$. Thus, the qualitative conclusion of the DWBA calculation is that the diffuse scattering provides a measure of $C(x, y)$, the height-height correlation function within the rough surface. 
It is worth noting that we would have reached the same qualitative conclusion within the Born approximation. In this case, however, $\mathrm{qz}_{\mathrm{z}}^{\mathrm{t}}$ in eqn (5) would have been replaced by $\mathrm{qz}_{\mathrm{z}}$ because refractive corrections are ignored in the Born approximation. The first factor in eqn (5) would then have been recognisable as the standard Debye-Waller factor. If the roughness amplitude were small enough to justify a first order expansion of the exponential term in the integrand of eqn (5), $S(q)$ would have, in the Born approximation, the traditional form we are all familiar with in neutron diffraction or small angle scattering.

Although one can think of a variety of different expressions to describe the height-height correlation function of a rough surface, $\mathrm{C}(\mathrm{x}, \mathrm{y})$, all of them have the property of falling off with distance in the reflecting plane in a fairly monotonic fashion. This means that the structure factor, $S(\mathbf{q})$, is a bell-shaped curve in both $q_{x}$ and $q_{y}$. In most reflectometry experiments, an integral of the diffuse scattering over $q_{y}$ (the coordinate into the plane of the paper in Figure 1) is performed automatically by the detector system and one only records the variation of the scattering as a function of $\mathrm{q}_{\mathrm{x}}$ and $\mathrm{q}_{\mathrm{z}}$.

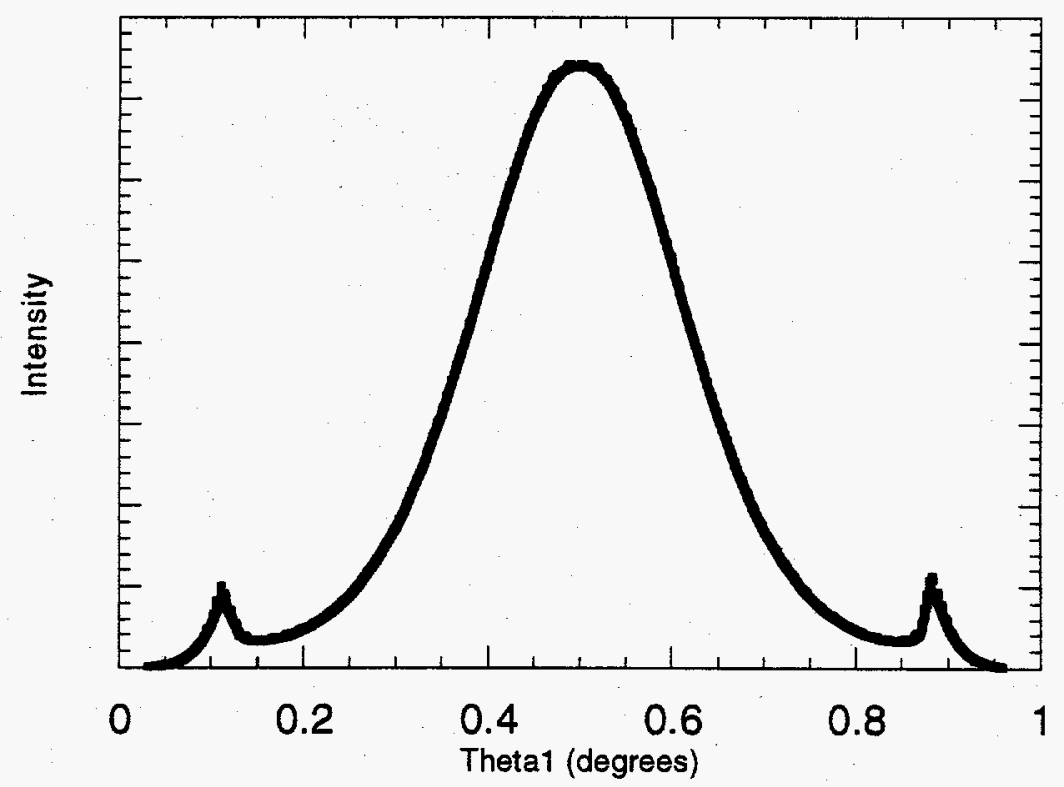

Figure 2a: Diffuse scattering for a rough sapphire surface with $\sigma=50 \AA$ at a scattering angle $\left(\theta_{1}+\theta_{2}\right)$ of $1^{\circ}$. The solid and dashed lines, which are essentially indistinguishable, are obtained when the transmission functions in eqn (4) are evaluated for smooth and rough surfaces respectively. The model for $C(x, y)$ used is that proposed by Sinha et al [4] with $h=0.4$ and $x=3000 \AA$. 


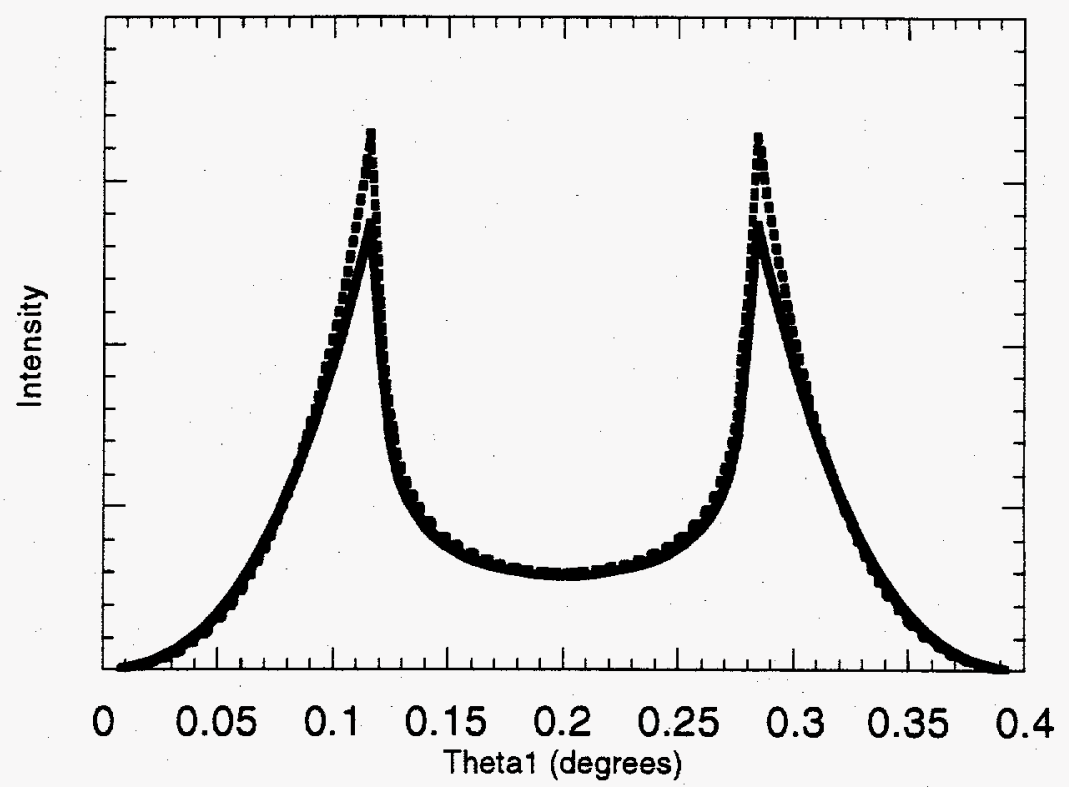

Figure 2b: Same as Figure 2a except that the scattering angle is $0.4^{\circ}$. In this Figure the difference between results obtained with transmission functions for rough (dashed line) and smooth surfaces (solid line) is much clearer.

Even though S(q) is bell-shaped, the measured intensity is not, as the solid lines in both parts of Figure 2 demonstrate. The two cusps in these Figures are a result of the structure of the Fresnel transmission functions $T_{i}(k)$ that appear in eqn (4) - these functions rise from a value of zero at $k=0$, to a peak value of 2 at the critical wavevector, and then decrease to an asymptotic value of unity at large values of $k$. The cusps in the figure are referred to as Yoneda scattering or angels' wings. Figure 2 also demonstrates that the observed shape of the diffuse scattering depends on $\mathrm{q}_{\mathrm{z}}$ : at small values of this parameter (cf Figure $2 \mathrm{~b}$ ), there may be no peak in the diffuse scattering at the specular condition $\mathrm{q}_{\mathrm{x}}=0$ (i.e. for $\theta_{1}=\theta_{2}$ ) whereas, at larger values of $q_{z}$ (corresponding to larger values of $\theta_{1}+\theta_{2}$ ) such a peak is evident. (cf Figure 2a). Once again, this results from the structure of the Fresnel transmission function.

It is clear that if we are to extract meaningful results for the height-height correlations from experimental data we need to be sure that eqn (4) is an accurate representation.

Unfortunately, we are not as sure as we would like to be, for several reasons. It turns out that the heuristic version of the DWBA presented above is not the preferred version $[5,6]$. Instead of using wavefunctions for the ideal surface to evaluate the matrix element in eqn (2), one ought to replace one of the wavefunctions by that for the rough surface. This is easy to fix as far as the notation is concerned - we simply change the subscript $i$ on one of the transmission functions to an $r$, implying that it is to be evaluated for the rough surface. Unfortunately, this leads to a result which violates time reversal symmetry [6]. Another version of the DWBA [7] replaces both of the transmission functions in eqn (4) by rough surface versions. However, as the dashed curves in Figure $2 \mathrm{~b}$ shows, this can give significantly different results from eqn (4) even when the same form for $S(q)$ is used. Detlef Bahr [8] has fitted several of the suggested forms of the cross section to X-ray data 
obtained by Weber and Lengeler [7] and has reached the conclusion that eqn (4) gives the

best result. However, it is still somewhat disquieting that extraction of quantitative values of $S(\mathbf{q})$ from diffuse scattering data has such an uncertain theoretical basis.

\section{Displaying Diffuse Scattering Data}

The most straightforward way to measure neutron reflectivity, including the diffuse scattering, is to use the time-of-flight (TOF) method with a linear position-sensitive detector either at a reactor or a pulsed spallation source. This method keeps the sample geometry constant and accumulates data for the entire reflection curve at the same time - low $-q_{z}$ reflectivity is measured with longwavelength neutrons while high- $\mathrm{q}_{\mathrm{z}}$ reflectivity is measured with short wavelengths. Because the decrease of reflectivity at high values of $\mathrm{q}_{z}$ is compensated to some extent by the increase of incident neutron intensity at short wavelengths, the entire reflectivity curve is measured with similar statistical accuracy. The natural way to present the data from such TOF reflectometers is as a plot spanned by the scattering angle $\theta_{2}$ and the neutron wavelength, $\lambda$. Such a plot is shown in Figure $3 a$. Many of our colleagues oppose the use of $\left(\lambda, \theta_{2}\right)$ plots, pointing out, quite correctly, that information about $\mathbf{S}(\mathbf{q})$ is more naturally displayed in the $\left(\mathrm{q}_{\mathrm{x}}, \mathrm{q}_{\mathrm{z}}\right)$ coordinate system. However, this argument ignores the fact that in eqn (4) the Fresnel transmission functions depend either on $\mathrm{k}_{1 \mathrm{z}}$ or $\mathrm{k}_{2 \mathrm{z}}$ and that structure related to these functions (such as Yoneda scattering) appears as straight fringes in a $\left(\lambda, \theta_{2}\right)$ plot (because $k_{2 z}=2 \pi \sin \theta_{2} / \lambda \approx$ $\left.2 \pi \theta_{2} / \lambda\right)$. Of course, $\left(\lambda, \theta_{2}\right)$ plots are easily transformed to $\left(\mathrm{q}_{\mathrm{x}}, \mathrm{q}_{\mathrm{z}}\right)$ plots, as Figure $3 \mathrm{~b}$ shows, but the two types of plot often highlight different physics.

\section{Diffuse Scattering from Films and Multilayers}

The data displayed in Figure 3 was obtained with a thin film of titanium on a sapphire substrate. In Figure 3a, we can easily distinguish the fringe due to the Yoneda scattering as the one that extrapolates to the origin of the plot. The other fringes in Figure $3 a$ (the ones closer to the left side of the figure), which extrapolate to $\theta_{2}=-\theta_{1}$ at $\lambda=0$, transform into fringes at constant $\mathrm{q}_{\mathrm{z}}$ in Figure $3 \mathrm{~b}$. These fringes arise because the roughness at the titanium/sapphire interface is correlated with that at the air/titanium interface [6] - that is, mountains at the titanium/air interface occur above mountains in the sapphire/titanium interface and valleys occur above valleys. The correlation does not have to be perfect i.e. the two interfaces do not need to be conformal - to generate such constant- $\mathrm{q}_{\mathrm{z}}$ fringes, but some degree of correlation must exist for these fringes to appear.

Figure 3: Total scattering from a thin layer of titanium on sapphire. Part (a) is plotted in a coordinate system spanned by $\lambda$ (abscissa) and $\theta_{2}$ (ordinate) while in part (b) the scattering has been transformed into 
the $q_{x}-q_{z}$ coordinate system. In part (a) specular scattering occurs along a horizontal line at $\theta_{1}=\theta_{2} \approx 1^{\circ}$ while in part (b) specular scattering corresponds to $q_{x}=0$. These data were recorded with the SPEAR reflectometer at LANSCE.

While it is straightforward to write down a closed expression for the diffuse scattering from a single monolayer on a substrate [6], there are no such compact expressions for multilayers. Nevertheless, calculations of this type can be done and, indeed, often successfully reproduce the qualitative features of experimental data [9]. In principal, one should be able to fit models to such data and deduce both the in-plane height-height correlation function and the way in which roughness correlations propagate from one layer to the next. However, to the knowledge of the authors, no such detailed study has yet been undertaken for multilayers.

\section{Limitations of the DWBA}

There are several situations in which one might expect the DWBA to give incorrect results. Since the calculation is based on second order perturbation theory, it will be inadequate when the perturbative term becomes so large that the third order term needs to be considered. De Boer [10] has considered this situation and finds that the DWBA will break down when

$$
\frac{\xi_{k_{1 z}^{2}}^{2}}{\left|k_{1}\right|}>1
$$

where $\xi$ is the characteristic distance over which $\mathrm{C}(\mathrm{x}, \mathrm{y})$ decays in the $\mathrm{x}-\mathrm{y}$ plane. Since $\theta_{1}$ is typically abut $1^{\circ}$, eqn (8) implies that the DWBA will break down when $\xi$ is much greater than about 500 times the neutron wavelength used for an experiment. This means that the $x$ or y dimension of a "typical" rough feature will have to be a micron or more for the DWBA to break down because second-order perturbation theory becomes inadequate. Of course, there may well be rough features that are smaller, but such a large value of $\xi$ can only be obtained if large features are present over the entire surface. Figure 4 illustrates what we have in mind. Even though the surface is rough on the Angstrøm scale, height correlations exist over micron distances because the surface seems to be composed of a series of rough facets whose smooth counterparts are the dashed lines in Figure 4.

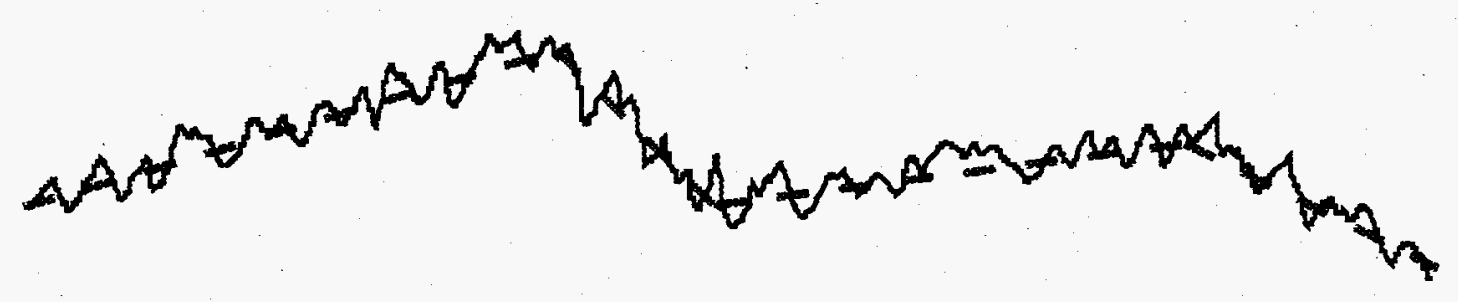

Figure 4: Sketch of a rough faceted surface. In addition to the high-frequency roughness, much larger facets, represented by the dashed lines, are also visible. The DWBA may fail for such surfaces.

In addition to the inherent limitation of the DWBA as a perturbation theory, the equations given above also make use of the continuity of the wavefunctions at the reflecting surface. In eqn (2), for example, the wavefunction above the surface has been replaced by an analytic continuation of the subsurface wavefunction. This replacement is adequate 
provided the amplitude of the surface roughness is not too great i.e. provide the subsurface wavefunction does not need to be extrapolated over too large a distance in the z-direction. It is straightforward to show that this means in practice that $\sigma$ should be less than about $1 /(10 \sqrt{\mathrm{Nb}})$, where $\mathrm{Nb}$ is the scattering length density of the reflecting medium. For most materials, this means that $\sigma$ must be less than about $50 \AA$ for the DWBA to work.

\section{Length scales probed by surface diffuse scattering}

It is instructive to think about the length scales within a reflecting surface that are probed by diffuse scattering. $\mathrm{q}_{\mathrm{x}}$ is given by

$$
\mathrm{q}_{\mathrm{x}}=\frac{2 \pi}{\lambda}\left(\cos \theta_{1}-\cos \theta_{2}\right) \approx \frac{\pi}{\lambda}\left(\theta_{2}^{2}-\theta_{1}^{2}\right)
$$

For reasonable parameter values (e.g. $\theta_{1}=0.02, \lambda=4 \AA$ and $\theta_{1}-\theta_{2}=0.001$ ), this means that $\mathrm{q}_{\mathrm{x}}$ can be as small as $10^{-5} \AA^{-1}$, corresponding to a length scale in the $\mathrm{x}-\mathrm{y}$ plane of 10 $\mu \mathrm{m}$ or more. This, of course, is a length scale that can easily be probed by optical microscopy. Thus, diffuse reflection from surfaces is one of the rare cases in which neutrons "see" the same length scales as light.

One can follow up on this observation and try to measure the diffuse neutron reflection from an optical grating (an experiment that was first done with $\mathrm{x}$-rays early in this century). Figure 5 shows the result of such an experiment conducted with a holographic grating composed of a patterned polymer film on a glass substrate. The grating used had 2800 lines per mm or a wavelength of $3571 \AA$. When we first did this experiment we did not think too hard and positioned the grating in the traditional orientation with the rulings perpendicular to the incident beam. We saw nothing! A little thought shows that the reason for this was that the rulings are too close together to produce a pattern at values of $\mathrm{q}_{\mathrm{x}}$ which were on our detector. Only by turning the grating so that the rulings were almost parallel to the neutron beam (the rulings made an angle of $1.7^{\circ}$ to the neutron beam) were we able to obtain the pattern shown in Figure 5.

Figure 5: Total neutron scattering from a holographic grating with an inter-line spacing of $3571 \AA$. Part (a) is a lambda-theta plot while part (b) shows the same data transformed to $\mathrm{q}_{\mathrm{X}}-\mathrm{q}_{\mathrm{Z}}$.

Figure $5 \mathrm{~b}$ shows that the principal features of the diffuse scattering do indeed occur at $\mathrm{q}_{\mathrm{x}} \approx \pm 0.000035 \AA^{-1}$ as would be expected for a grating of period $3571 \AA$ at an angle of $1.7^{\circ}$ to the neutron beam. It is also clear from Figure 5 that the peaks in the diffuse scattering occur at a value of $\mathrm{q}_{z}$ of about $0.013 \AA^{-1}$ and that there is a fringe in the specular scattering at this same value of $\mathrm{qz}_{\mathrm{z}}$. Detailed examination of the specular scattering reveals that it derives from a layer about $600 \AA$ thick on the glass substrate - the polymethyl 
methacrylate coating on which the holographic pattern is written. The fact that the diffuse and specular scattering both show oscillating dependence on $\mathrm{q}_{\mathrm{z}}$ is another manifestation of the fact that the diffuse scattering involves products of transmission functions (cf eqn 4 ) in addition to the fluctuation spectrum of the rough surface. In this case, the transmission functions are those for a film on a substrate rather than for the rough surface alone [11]. Like the corresponding reflectivities, such transmission functions display oscillating structure due to the interference between scattering from the two film surfaces.

An example of the range of surface length scales that can be probed by diffuse neutron reflection is shown in Figure 6 . The first part of this Figure is a density plot of the total scattering from a thick multi-bilayer of dimyristoyl phosphatidylcholine (DMPC) spun on to a silicon substrate and maintained in a humid $\mathrm{D}_{2} \mathrm{O}$ atmosphere at room temperature. There is a fringe of diffuse scattering which passes through the Bragg peak from the multibilayer structure that is seen on the far left of the picture at theta $=0.5^{\circ}$. The fact that this fringe extrapolates to $\theta_{2}=-\theta_{1}$ at $\lambda=0$ indicates that it is one of the constant- $q_{z}$ fringes described earlier that result from correlated roughness of the interfaces within the multibilayer structure.

Figure 6: Part (a) shows the total scattering from a DMPC multi-bilayer structure. Part (b) is the profile of the scattering along the diffuse fringe, plotted as a function of $q_{x}$.

Unfortunately, our position-sensitive detector was not long enough to record the entire fringe in Figure $6 \mathrm{a}$ (in fact, we would have needed a 0.8 metre long detector to do so!). However, the SPEAR reflectometer at LANSCE is built so that the distance between the sample and the detector can be changed continuously from about $50 \mathrm{~cm}$ to more than $5 \mathrm{~m}$. The height of the detector above the reflecting plane can also be continuously varied over a $50 \mathrm{~cm}$ range. This flexibility allowed us to follow the fringe in Figure $6 \mathrm{a}$ out to a scattering angle of about $12.5^{\circ}$. The variation of the scattered intensity along the fringe is shown in Figure $6 \mathrm{~b}$. A remarkable thing about this figure is the range of $\mathrm{q}_{\mathrm{x}}$ probed - from about $0.0002 \AA^{-1}$ to about $0.011 \AA^{-1}$ — corresponding to length scales ranging from about $500 \AA$ to over $3 \mu \mathrm{m}$. Figure $6 \mathrm{~b}$ is also quite different from the usual bell-shaped form of $S(q)$ that peaks at $\mathrm{q}_{\mathrm{x}}=0$. A detailed analysis of this profile and its implications for the structure of lipid layers is still in progress.

\section{Diffuse scattering from faceted surfaces}

Figure $6 \mathrm{a}$ is also interesting because it displays a feature that we have not yet encountered. In addition to the standard Yoneda fringe that extends from the critical wavelength in the specular scattering towards the origin of Figure 6a, there also appears to be another fringe 
which extrapolates to a negative value of $\theta_{2}$ at $\lambda=0$. A similar phenomenon is evident in Figure $7 \mathrm{~b}$ which shows scattering from a planar grain boundary between nickel and a nullmatrix ${ }^{60} \mathrm{Ni}$-enriched material [12]. Figure 7a shows a case in which the "extra" fringe occurs in the absence of Yoneda scattering for silicon wafer sample. In both parts of Figure 7 it is fairly clear that the extra fringe extrapolates to $\theta_{2}=-\theta_{1}$ at $\lambda=0$ and is therefore characterised by $\mathrm{q}_{\mathrm{z}}=$ constant. With a little imagination, one can postulate that the "extra" fringe in Figure $6 \mathrm{a}$ also extrapolates to $-\theta_{1}$.

Figure 7: Grey scale plots of specular plus diffuse scattering as a function of $\theta_{2}$ and $\lambda$. Part (a): a polished silicon wafer; part (b) a planar grain boundary between natural nickel and a null-matrix $60 \mathrm{Ni}$-enriched material.

For a long time we were very puzzled by these extra, constant- $\mathrm{q}_{\mathrm{z}}$ fringes. Even more puzzling was the fact that in some case we observed the extra fringe in the absence of Yoneda scattering, in spite of the fact that the DWBA tells us that we must always see Yoneda wings with any rough surface. We wondered whether the constancy of $\mathrm{q}_{\mathrm{z}}$ along the extra fringe might indicate the presence of some length scale perpendicular to the surface. If this were the case, the controlling parameter ought to be the value of $\mathrm{q}_{\mathrm{z}}$ within the reflecting medium, which takes a complex value along the entire fringe except at $\mathrm{q}_{\mathrm{x}}=0$. We were unable to imagine what the corresponding length scale might be. Clearly, since we do not have multiple interfaces in the systems that gave rise to the patterns in Figure 7, it is impossible to attribute the extra fringe to correlated roughness as we asserted for Figure 3.

The resolution of the paradox turns out to be contained in Figure 4. Imagine for a moment that the surface is described by the dashed line in Figure 4, i.e. that the surface is composed of an assembly of smooth facets. If these facets are large enough, the neutron wavefunction close to the surface will not look much like that for the average surface. Rather, we might expect that each facet would reflect specularly and more or less independently of the other facets. It is fairly straightforward to evaluate what the scattering would look like in such a case [13]. It is even easier to understand qualitatively how such a surface may yield an apparent fringe at constant $\mathrm{q}_{\mathrm{z}}$ that extends from the critical edge in the specular scattering. 


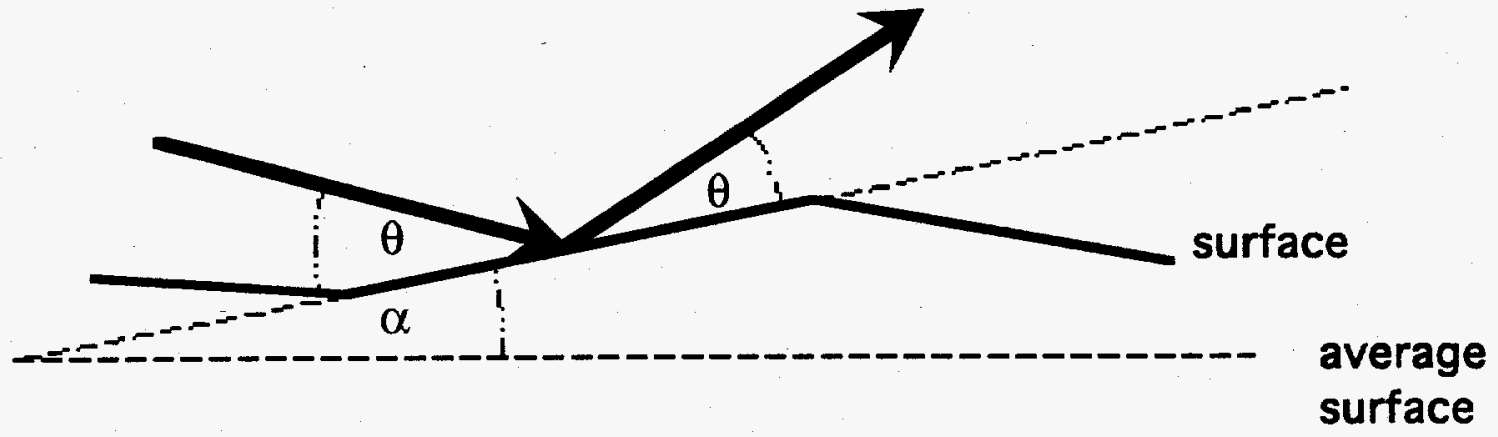

Figure 8: Scattering from a faceted surface.

As shown in Figure 8, the angles of incidence and reflection measured with respect to the reflecting facet are equal because the scattering is assumed to be specular. The apparent value of $\mathrm{q}_{\mathrm{z}}$, obtained by measuring the angles of incidence and reflection with respect to the average surface is given by:

$$
\mathrm{q}_{\mathrm{z}}^{\text {apparent }}=\frac{2 \pi}{\lambda}(\sin (\theta-\alpha)+\sin (\theta+\alpha)) \approx \frac{2 \pi}{\lambda} 2 \sin \theta
$$

i.e. the apparent value of $\mathrm{q}_{\mathrm{z}}$ is just twice the component of the neutron wavevector perpendicular to the facet. This means that any feature of the specular scattering - the critical edge, for example - will map to an apparently constant value of $\mathrm{q}_{\mathrm{z}}$. The extra fringe we see in Figures 6 and 7 is just the locus of the critical edge for radiation specularly reflected by the surface facets.

This explanation is made even more plausible by the results presented in Figure 9, which shows cuts through the data of Figure 7 a for various values of $\theta_{2}$ as a function of $\mathrm{q}_{\mathrm{z}}$. Each of the cuts has the same shape as the specular scattering, as one would expect if the explanation described above were correct.

Figure 9: Cuts through the data of Figure $7 \mathrm{a}$ at $\theta_{2}=0.6^{\circ}$ (specular), $0.45^{\circ}, 0.39^{\circ}$ and $0.23^{\circ}$ as a function of $q_{z}$.

In light of the explanation presented above for the extra fringe, it is not surprising that it is observed in Figure $6 \mathrm{a}$, since we already know from Figure $6 \mathrm{~b}$ that the interfaces between lipid bilayers are rough on length scales up to several microns i.e. that these interfaces have facet-like features. One problem that this brings is that high-frequency and low-frequency (facets) roughness have different effects on the specular scattering. In a seminal paper, Nevot and Croce [14] showed that the specular reflectivity of a rough surface is related to that for a smooth surface by the equation: 


$$
\mathrm{R}_{\mathrm{r}}=\mathrm{R}_{\mathrm{i}} \exp \left(-\mathrm{q}_{\mathrm{z}} \mathrm{q}_{\mathrm{z}}^{\mathrm{t}} \sigma^{2} / 2\right)
$$

This equation was derived under the assumption of high frequency roughness and can also be obtained from the DWBA if one is careful [6]. The expression has been verified numerous times in the past decade and is now used almost universally to fit specular reflectivities both in neutron and x-ray reflectivity experiments.

For surfaces with smooth facets, the reduction of the average-surface reflectivity caused by the facets depends very much on the facet size. If this size is larger than the distance on the surface over which the radiation averages, one obtains the Debye Waller form given by Beckmann and Spizzichino [2] for the reflection of radar waves from rough terrain:

$$
R_{r}=R_{i} \exp \left(-q_{z}^{2} \sigma^{2} / 2\right)
$$

To the authors' knowledge, the detailed effect of the spatial frequency of the roughness on the neutron or $\mathrm{x}$-ray reflectivity of a surface has not yet been investigated in detail, although this is a prerequisite for obtaining accurate information about surface roughness from reflectivity experiments.

\section{Conclusion}

In this paper, we have given a brief introduction to the current state of knowledge about the effect of surface and interface roughness on neutron reflectivity experiments. In some cases, notably where the roughness has a high spatial frequency and is of small amplitude, a detailed theoretical description of the diffuse scattering and the effect of roughness on specular scattering can be obtained from the distorted wave Born approximation. In these cases, experimental data can be analysed to provide a quantitative description of the fluctuation spectrum of surface roughness. When the spatial frequency of the roughness is smaller, or when both high and low frequencies are present, the situation is less clear and present theory only permits qualitative deductions from the experimental data.

\section{Acknowledgments}

This work was funded by the Office of Basic Energy Sciences of the U.S. Department of Energy under contract W-7405-ENG-36 with the University of California.

\section{References}

[1] W. A. Hamilton, P. D. Butler, S. M. Baker, G. S. Smith, John B. Hayter, L. J. Magid, and R. Pynn, Phys. Rev. Lett. 72, 2219 (1994)

[2] P. Beckmann and A Spizzichino, The Scattering of Electromagnetic Waves from Rough Surfaces (Artech House, Norwood, MA, 1987)

[3] C. Majkrzak, This Volume

[4] S. K. Sinha, E. B. Sirota, S. Garoff, and H. B. Stanley, Phys. Rev. B 38, 2297 (1988)

[5] A. Messiah, Mecanique Quantique, Tome II (Dunod, Paris, 1960)

[6] R. Pynn, Phys. Rev. B 45, 602 (1992)

[7] W. Weber and B. Lengeler, Phys. Rev. B 46, 7593 (1992)

[8] D. Bahr, private communication 
[9] LANSCE Newsletter number 14 (published by Los Alamos National Laboratory)

[10] D. K. G. de Boer, Phys. Rev. B 49, 5817 (1994)

[11] R. Pynn, SPIE Vol 1738, p 270 (1992)

[12] M. Fitzsimmons, to be published

[13] R. Pynn and S. M. Baker, Physica B 198, 1, (1994)

[14] L. Nevot and P. Croce, Rev. Phys. Appl. 15, 761 (1980); P. Croce and L. Nevot, Rev. Phys. Appl. 11, 113, (1976) 


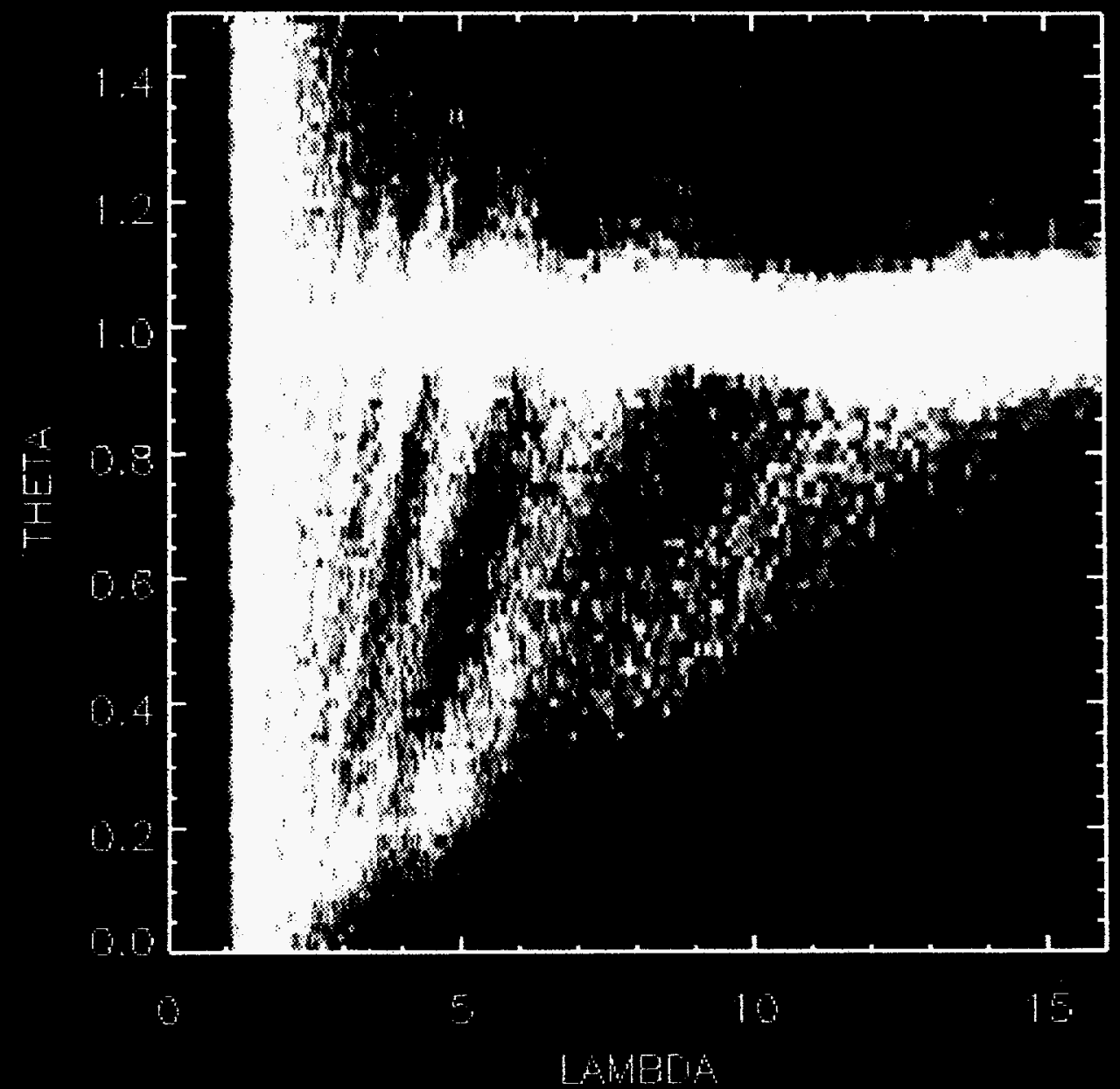

Fig. $3 a$ 


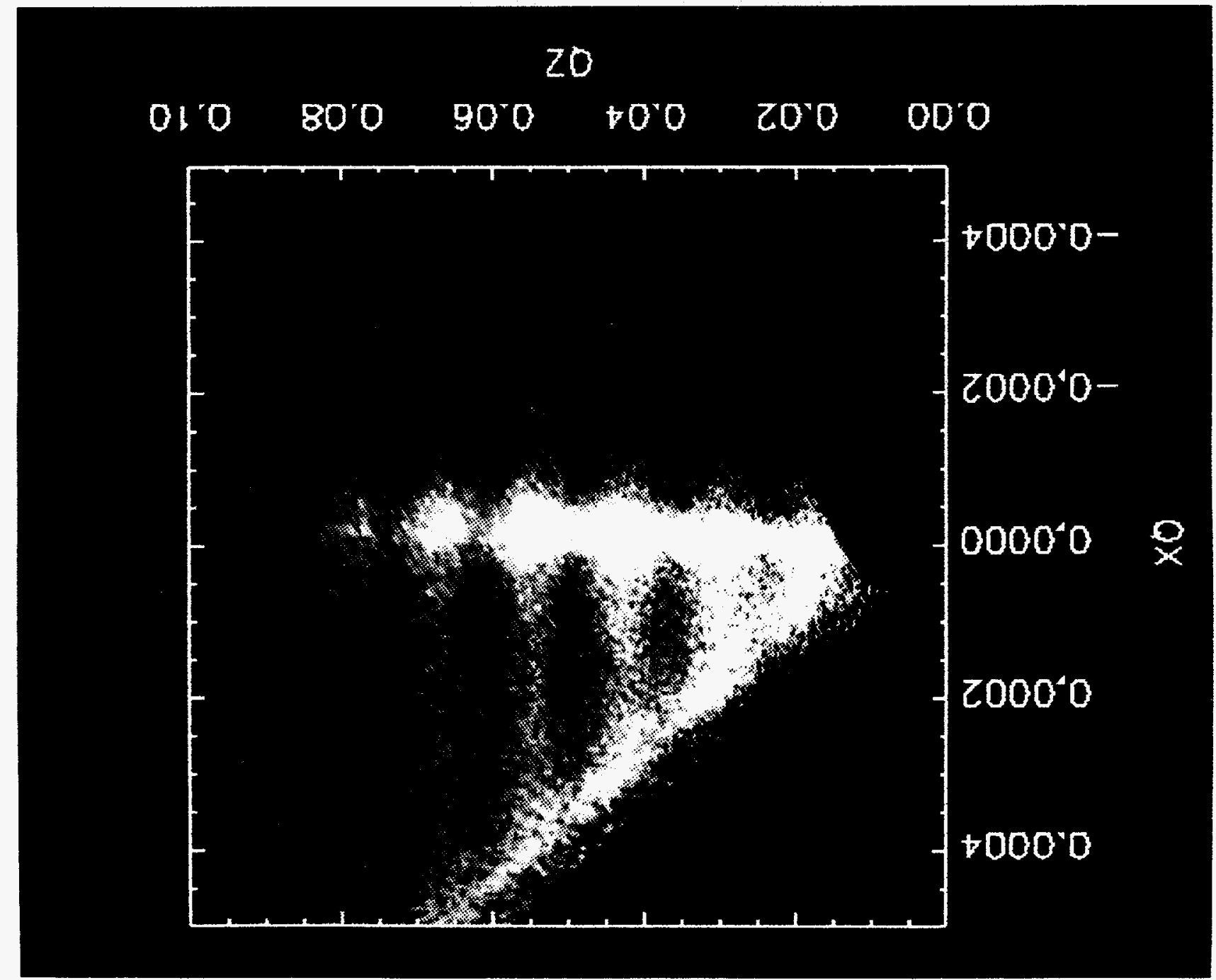




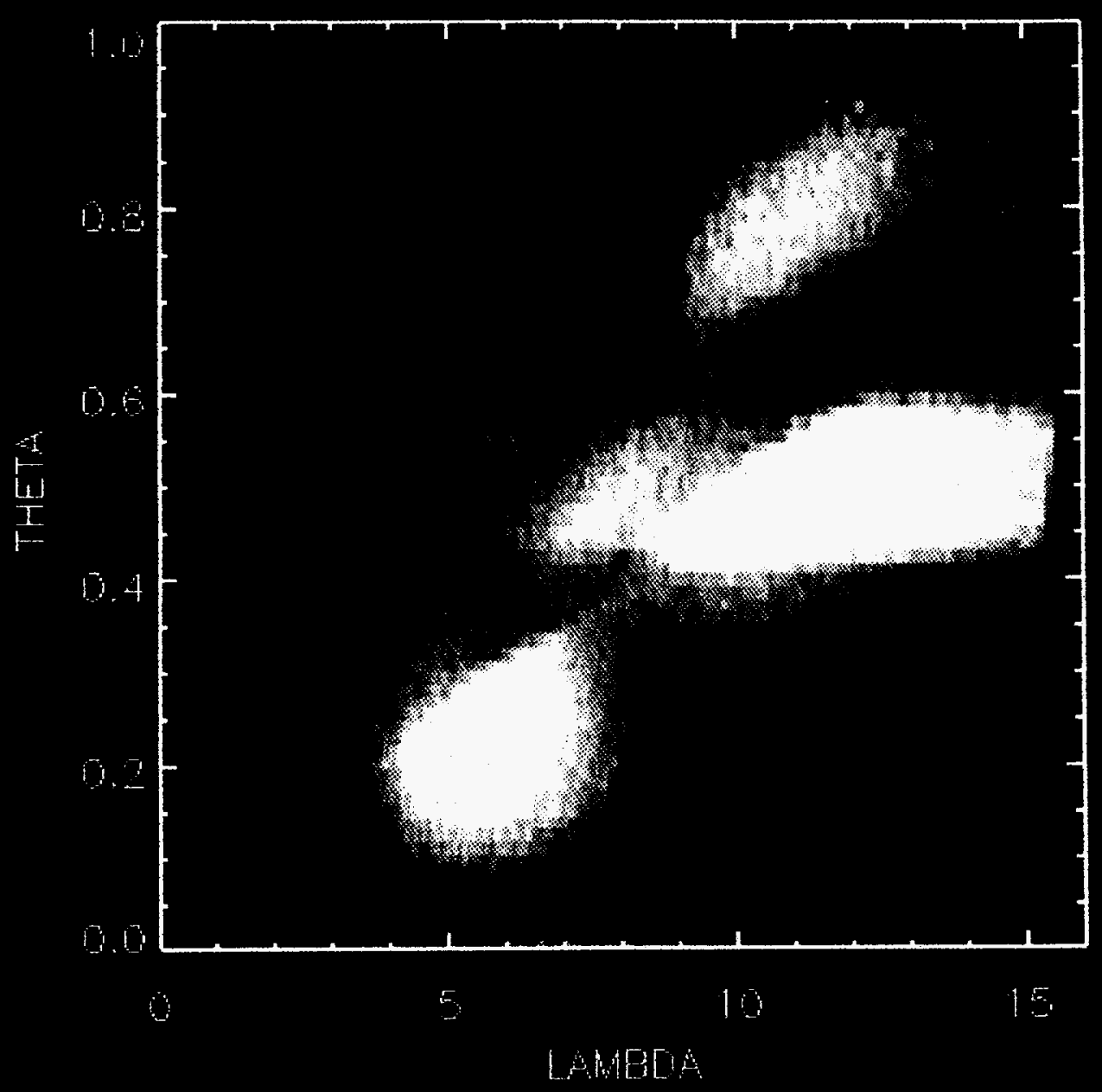

Fig. 5a 


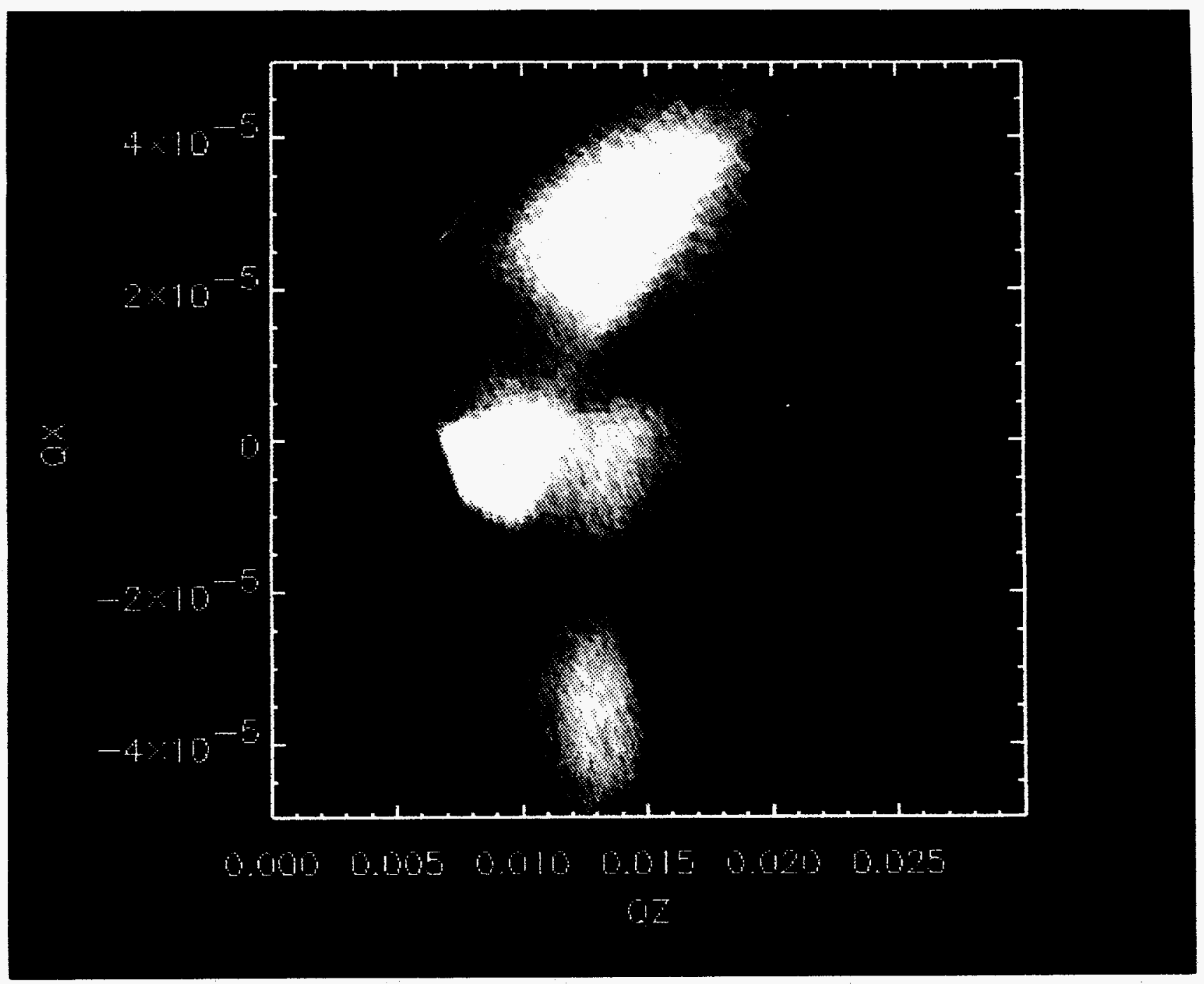

Fig. 5b 


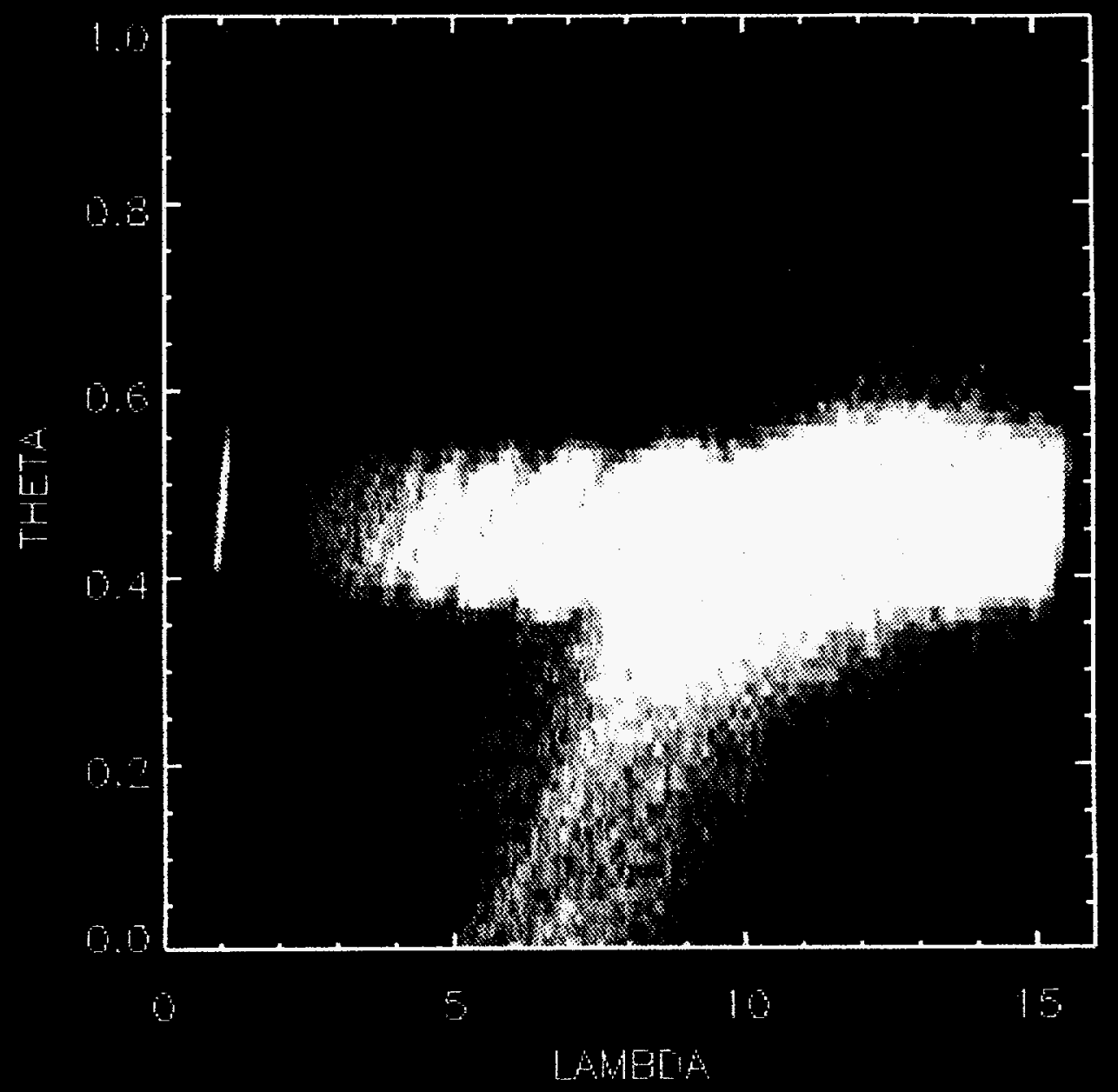

Fig. 6a 


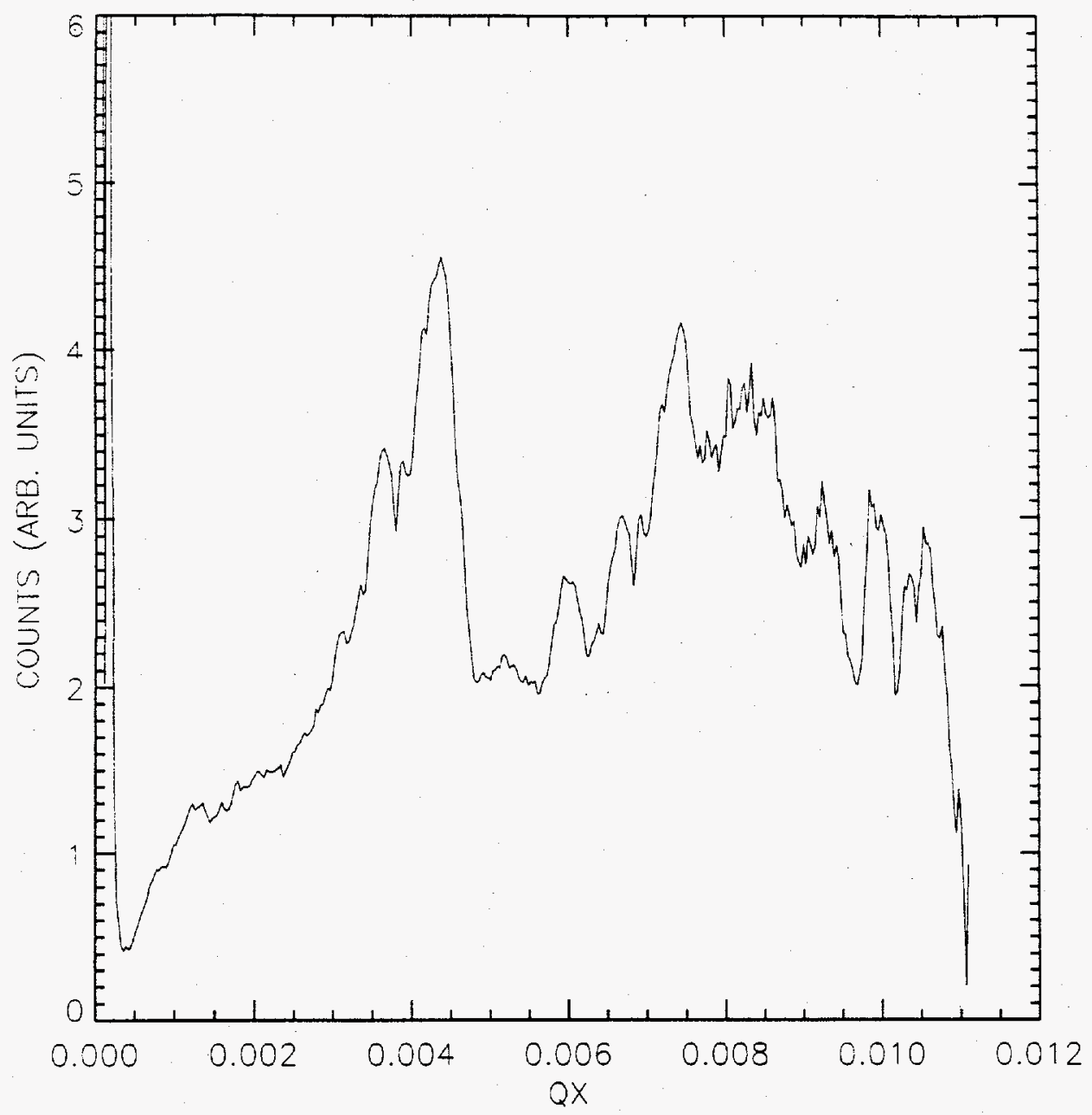

Fig. 6b 


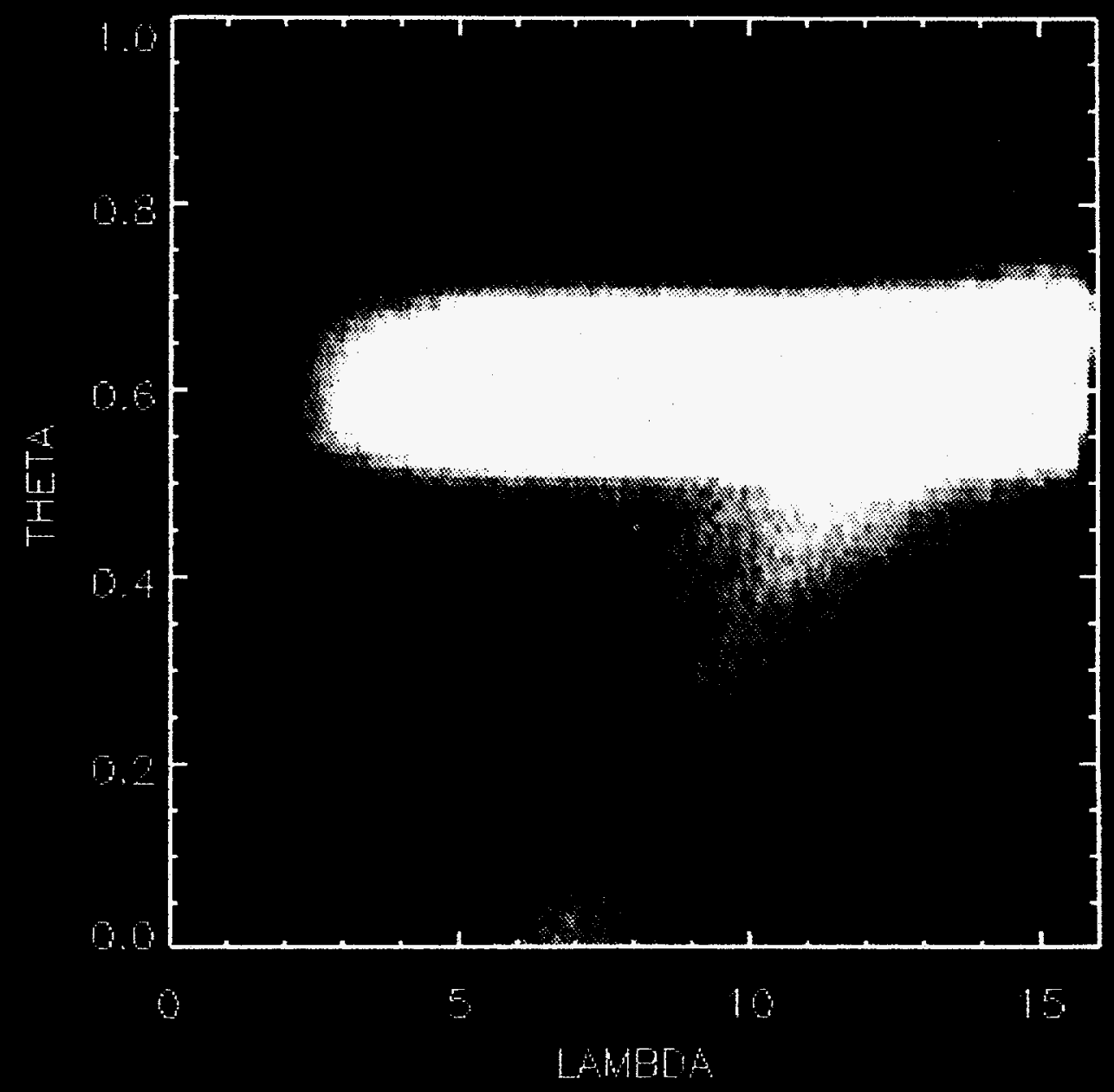

Fig. $7 a$ 


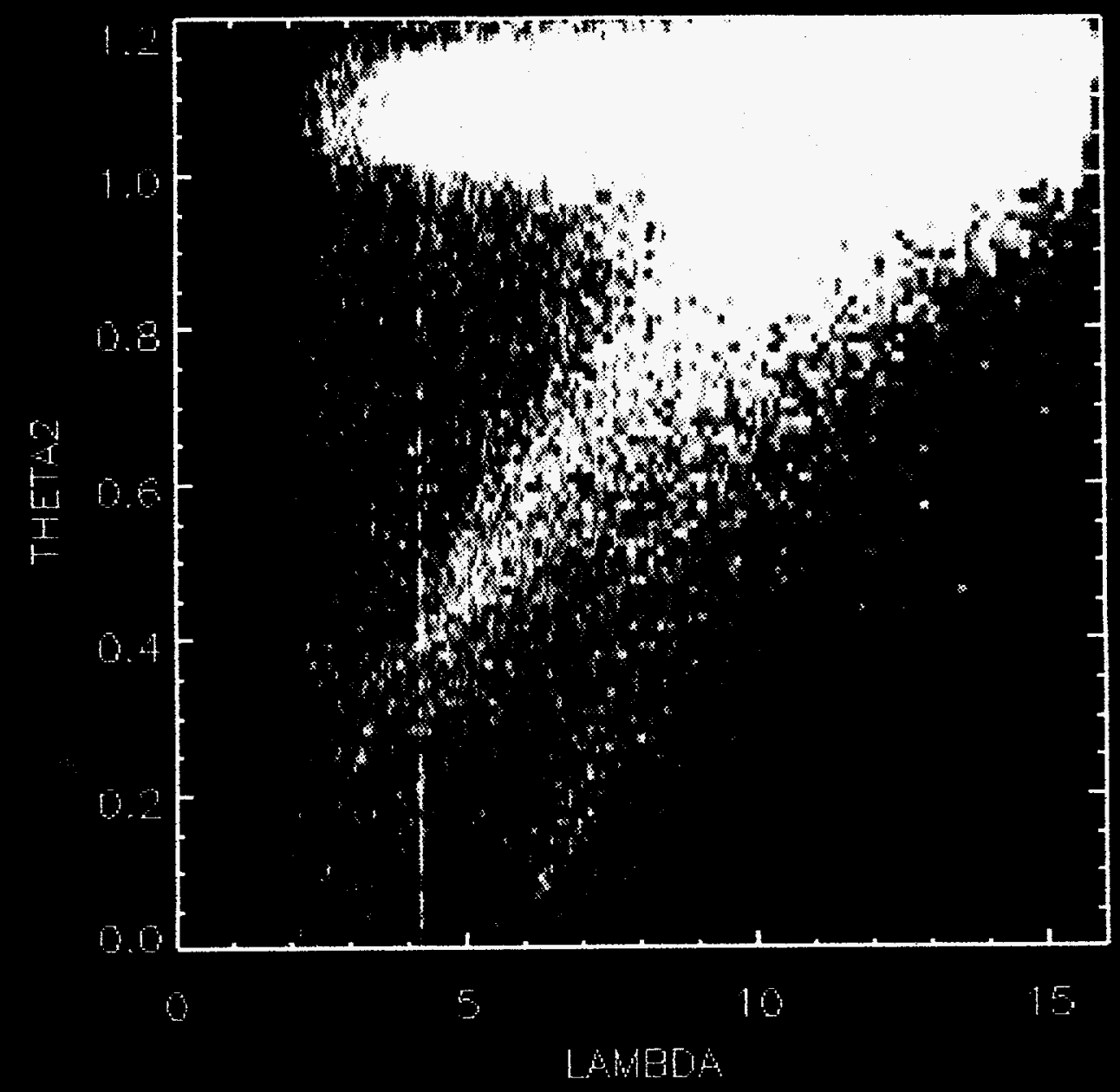

Fig. $7 b$ 


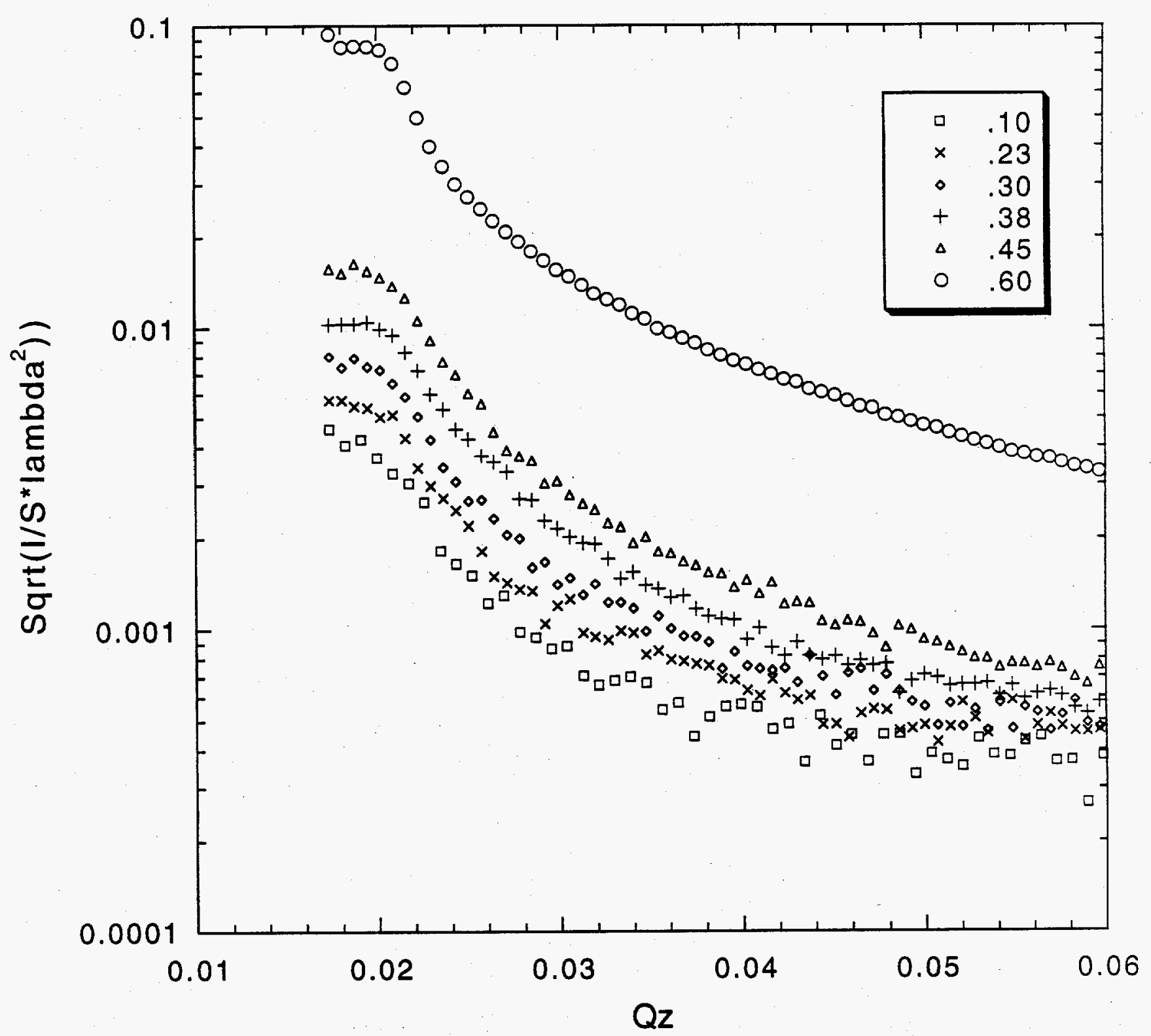

Fig. 9 